\title{
Nursing Standard
}

\section{infection/how to series}

\section{Why you should read this article:}

- To optimise your practice when undertaking temperature measurements at various sites

- To understand the importance of measuring temperature to provide information about a patient's health and its role in diagnosis and patient monitoring

- To enhance your knowledge of the factors that can influence the accuracy of temperature measurements

\section{How to measure a patient's temperature non-invasively}

Joyce Smith, Melanie Ann Rushton, Mike Barker

Citation

Smith J, Rushton MA, Barker M (2019) How to measure a patient's temperature non-invasively. Nursing Standard. doi: 10.7748/ns.2019.e11346

Peer review

This article has been subject to external double-blind peer review and checked for plagiarism using automated software

Correspondence

J.Smith2@salford.ac.uk

Conflict of interest

None declared

Accepted

18 February 2019

Published online

XXXXX

Disclaimer

Please note that information provided by Nursing Standard is not sufficient to make the reader competent to perform the task. All clinical skills should be formally assessed at the bedside by a nurse educator or mentor. It is the nurse's responsibility to ensure their practice remains up to date and reflects the latest evidence

\section{Rationale and key points}

Temperature is a vital sign that is included in all early warning scoring tools and as part of patient observations. This article outlines the various methods that can be used to measure a patient's temperature accurately.

» Temperature is important in establishing a baseline to evaluate treatment, monitoring signs of any allergic reaction or infection, and recognising alterations in temperature, for example hypothermia and hyperthermia.

» Nurses should understand the pathophysiology of temperature regulation in addition to the environmental factors that may result in inaccurate measurements. They should also be familiar with the various methods used to measure temperature. 
"It is crucial to identify any signs of clinical deterioration, including elevated or lowered temperature, and respond to these in a timely manner.

\section{Reflective activity}

'How to' articles can assist you to update your knowledge to ensure evidence-based practice. Apply this article to your practice. Reflect on and write a short account of:

1. How reading this article will change your practice in measuring patients' temperature.

2. How this article could be used to provide information to patients about temperature measurements.

\section{Author details}

Joyce Smith, adult lecturer, School of Health and Society, University of Salford, Salford, England; Melanie Ann Rushton, adult lecturer, School of Health and Society, University of Salford, Salford, England; Mike Barker, adult lecturer, School of Health and Society, University of Salford, Salford, England

\section{Keywords}

body temperature, clinical skills, early warning scores, infection, nursing care, observations, sepsis, signs and symptoms

Key points

- Temperature measurements are an important vital sign that may be used by nurses to determine the degree of patient illness, as well as informing clinical decision-making during diagnosis and treatment

- Elevated temperature is a significant clinical indicator of illness, primarily associated with bacterial or viral infections, or inflammation. Conversely, a significantly lowered temperature can result in hypothermia. Therefore, it is important that nurses are able to undertake temperature measurement accurately

- Non-invasive methods for measuring temperature include the temporal, tympanic, axillary and oral sites

Temperature is one of the vital signs that provide an indication of the status of the body's essential functions, alongside pulse rate, respiration rate, oxygen saturations, level of consciousness and blood pressure (Royal College of Physicians (RCP) 2017). Body temperature is regulated by the hypothalamus using signals received from sensory neurons known as thermoreceptors, which are located in the skin (Grainger 2013). The normal body temperature range is between $36^{\circ} \mathrm{C}$ and $37.5^{\circ} \mathrm{C}$, and healthy individuals maintain a constant body temperature despite environmental variations.

Elevated temperature is a significant clinical indicator of illness, primarily associated with bacterial or viral infections, or inflammation. Conversely, a significantly lowered temperature can result in hypothermia. Therefore, it is important that nurses are able to undertake temperature measurement accurately. This article outlines the main non-invasive methods used to measure temperature.

\section{Preparation and equipment}

» Before undertaking a temperature measurement, the nurse should ensure that the clinical environment is safe so that any risk to themselves and the patient is minimised, for example by ensuring that there are no wires or equipment that may impede movement.

» Ensure that the patient's privacy and dignity is maintained, for example by closing the curtains around the bed area or finding a private room if necessary.

» Use the 'look, listen and feel' approach to initially assess the patient (Resuscitation Council (UK) 2019):

- Look at the patient's appearance, for example do they appear pale or flushed, or are they perspiring?

- Listen to the patient, for example do they sound breathless or confused?

- Feel the patient's skin: is it cool and 'clammy' or warm to the touch?

» Select the optimal site for measuring the patient's temperature. Non-invasive options include the tympanic (ear), oral, axillary (armpit) and temporal artery sites, while invasive options include the pulmonary artery, bladder and rectal sites. 
- The tympanic site is frequently used and might be more appropriate than other sites in some patients; for example, a patient who is breathless might not be able to tolerate an oral temperature measurement.

- The oral site is considered to accurately reflect core body temperature; however, it not suitable for patients who are on oxygen therapy, are confused, have difficulty breathing, have undergone facial surgery or are unconscious; in these cases, the tympanic or temporal artery sites would be more appropriate.

- The axillary site is not considered to be the most accurate site for recording a patient's body temperature; however, it can be used if other sites are not available or appropriate.

- The temporal artery site is considered the least invasive method of measuring temperature, and therefore may be useful in certain patients, for example infants and children.

» Ensure that the necessary equipment is accessible and in optimal working order, for example calibrated or cleaned in line with local policy. Depending on the selected site and method to be used to measure temperature, the necessary equipment may include:

- Single-use heat-sensitive chemical strip for use at the oral and axillary sites.

- Tympanic thermometer with disposable probe covers.

- Temporal artery thermometer - this may be a device that comes into contact with the skin on the forehead, or a noncontact infrared forehead scanner. Some models require the use of disposable probe covers.

- Alcohol wipes.

- Early warning scoring chart or patient observations chart.

- Clinical waste bin.

» Explain the procedure to the patient and obtain consent from them. Verbal consent is acceptable if the patient has the capacity to make an informed decision. If the patient appears anxious, provide reassurance by explaining that measuring temperature is a routine part of monitoring their physiological observations.

» Undertake effective hand hygiene (Hillier 2015) by washing your hands using bactericidal soap and water or bactericidal alcohol hand rub. Effective hand hygiene must also be undertaken after measuring the patient's temperature.

\section{Procedure}

\section{Tympanic temperature measurements}

The tympanic site is frequently used to measure temperature because the ear drum has the same blood supply as the hypothalamus, which means that the tympanic temperature is a direct reflection of the core body temperature (Chue et al 2012).

1. Visually inspect the ear canal. If the patient has a hearing aid, remove it and wait for ten minutes before undertaking the reading. Check that the ear canal is clear of any signs of wax, blood or foreign bodies.

2. For infection prevention and control purposes, place a disposable cover on the probe, ensuring that the cover fits correctly and that there are no wrinkles over the tip of the probe.

3. Gently pull the pinna (the outer visible part of the ear) up and back and carefully insert the probe into the patient's ear canal. Press the scan button. The thermometer will beep when the recording is complete.

4. Remove the probe from the patient's ear and safely dispose of the probe cover in a clinical waste bin.

5. Record the temperature reading on the patient's early warning scoring chart or patient observations chart, and escalate any concerns regarding any abnormalities.

6. Clean the tympanic thermometer in accordance with local policy and the manufacturer's guidelines.

\section{Oral or axillary temperature measurements}

Single-use heat-sensitive chemical strips are often used to measure temperature at the oral or axillary sites. The posterior sublingual area is used when measuring temperature orally, because this is close to the sublingual artery and is therefore considered to accurately reflect core body temperature. 
1. If using the oral site, ensure that the patient has not consumed a hot drink or hot food, or smoked before measuring their temperature.

2. Check that the single-use heat-sensitive chemical strip is within date and appropriate for single-use. Single-use thermometers are individually wrapped, so peel open one strip. Ensure that you do not touch the chemical dots and hold the other end of the strip.

3. - If using the oral site, ask patient to open their mouth and place the strip in the posterior sublingual pocket of tissue at the base of the patient's tongue for one minute. Ask the patient to press down with their tongue and keep their mouth closed.

- If using the axillary site, place the strip in the centre of the patient's armpit for three minutes, and ask the patient to keep their arm firmly pressed against the side of their chest throughout. The strip must be in contact with the patient's skin and not their clothing.

4. Remove the thermometer and wait ten seconds. Look at the dots to read the temperature; the last recorded blue dot will indicate the patient's temperature. Examples of temperature readings using a single-use heat-sensitive chemical strip are shown in Figure 1.

5. Record the temperature on the patient's early warning scoring chart or patient observations chart, and escalate any concerns regarding any abnormalities.

6. Dispose of the strip in a clinical waste bin.

\section{Figure 1. Examples of temperature readings using a single-use heat-sensitive chemical strip}

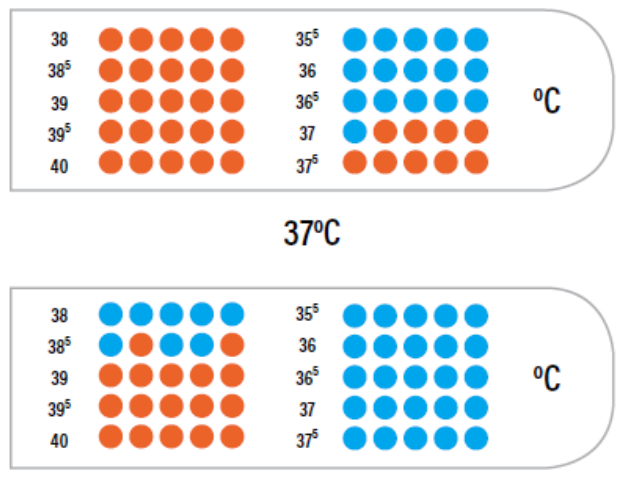

$38.8^{\circ} \mathrm{C}$

\section{Temporal artery temperature measurements}

[Q1. we have rephrased this section in accordance with your comments - please read through and confirm if you are happy with this, or indicate any changes required]

The temporal artery site is used to measure temperature at the temples on either side of the patient's forehead, which are close to major blood vessels. The patient's temperature can be measured in seconds using this site because of the proximity of the temporal artery. There are two types of temporal artery thermometers; one type involves contact with the skin on the forehead, while the other type is an infrared forehead scanner that does not contact the skin.

1. Move any hair covering the patient's forehead out of the way. Where possible, address any factors that could affect the accuracy of the reading, such as hats, wigs, bandages, sweating or if the environment is particularly hot or cold. Consider using another site to measure temperature if these factors cannot be addressed.

2. Check the lens of the temporal artery thermometer and clean it with an alcohol wipe. Some models will have a probe cover that should be removed before use, and some models will require a disposable cover to be placed over the lens, so follow the manufacturer's guidelines.

3. - If using a temporal artery thermometer that requires contact with the skin, place the probe on the centre of the patient's forehead. Press and hold down the scan button, and move the probe steadily either left or right across the patient's 
forehead and into their hairline. While still holding down the scan button, lift the probe and place it behind the earlobe; this is particularly important if the patient is sweating to ensure an accurate reading. Release the scan button and read the temperature [Q2. are you happy with the above description?].

- If using a non-contact infrared forehead scanner, point the scanner straight at the patient's forehead [Q3. would this be pointed at the centre of their forehead or at their temple on either side of the forehead?], holding it 3-5cm away from the skin [Q4. ok to add this?]. Press the scan button. [Q5. will the device beep to indicate when the recording is complete?].

4. Record the temperature on the patient's early warning scoring chart or patient observations chart, and escalate any concerns regarding any abnormalities.

5. Clean the temporal artery thermometer in accordance with local policy and the manufacturer's guidelines.

\section{Evidence base}

Thermoregulation is the process that regulates core body temperature and enables the body to maintain its core internal temperature. Thermoregulation has several contributory factors including cellular metabolism, exercise and ambient temperature (the temperature of the surrounding air). Elevated temperature can indicate illness such as bacterial or viral infections, or inflammation (Grainger 2013). A temperature of $37.5^{\circ} \mathrm{C}-40^{\circ} \mathrm{C}$ constitutes pyrexia (fever), which is caused for example by an elevated bacterial load in conditions such as influenza or sepsis, or meningitis in infants. Hyperpyrexia is a potentially fatal rise in body temperature above $40^{\circ} \mathrm{C}$, which may be caused for example by spinal cord injury, malaria or drug overdose (Grainger 2013). A significantly lowered temperature of under $35^{\circ} \mathrm{C}$ can result in hypothermia, a potentially fatal physiological state that affects frail older people in particular (Grainger 2013).

Temperature measurements are an important vital sign that may be used by nurses to determine the degree of patient illness, as well as informing clinical decision-making during diagnosis and treatment (Barnason et al 2012, Sund-Levander and Grodzinsky 2013). Accurate temperature recording is essential in establishing baseline measurements, which can provide vital information about a patient's health and is central to diagnosis and patient monitoring. According to the National Institute for Health and Care Excellence (NICE) (2007), temperature is one of the vital signs that should be used to recognise and respond to acute illness. Temperature is also one of the measurements that comprise the National Early Warning Scoring (NEWS) 2 (RCP 2017) tool, which was developed to identify and respond to clinical deterioration. Faulds and Meeking (2013) found that the thermoregulation of critically ill patients is often disrupted, which may be a consequence of a disease process such as fever. Therefore, close monitoring and regulation of body temperature is an important part of the care of critically ill patients.

Before selecting the optimal method to use to measure temperature, it is important to consider the patient's comfort, privacy and dignity, as well as the invasiveness of the procedure. Various invasive and non-invasive methods can be used to measure temperature. Invasive methods include pulmonary artery temperature, using a catheter to measure pulmonary artery temperature; rectal temperature, using a rectal thermometer; and bladder temperature, using a temperature-sensing indwelling urinary catheter to measure core body temperature (Barnason et al 2012). However, Sund-Levander and Grodzinsky (2013) emphasised that the rectal measurement of temperature is unhygienic and can pose a risk to patients by increasing physical and psychological stress, anxiety and discomfort. The pulmonary artery is considered the 'gold standard' site for measuring core body temperature (Carroll 2012); however, this is primarily undertaken in intensive care units.

Non-invasive methods for measuring temperature include using the temporal, tympanic, axillary and oral sites (Barnason et al 2012). Gasim et al (2013) found that measuring tympanic temperature was simple to undertake and provided a quick temperature reading. However, Davie and Amoore (2010) suggested that there is little consensus on the optimal method to use when measuring temperature. It is important to be aware that temperature measurements may vary slightly depending on the site used (Sund-Levander and Grodzinsky 2013). 
[Q6. we think it would be useful to add some of the factors that might influence readings that nurses should be aware of, for example time of day, environmental factors, the level of activity, medicines, emotions etc. Could you add a sentence listing some of these factors, supported by a reference?]

Practitioner error is an additional factor that should be considered by nurses when measuring patients' temperature. For example, Creagh-Brown et al (2005) found that nurses do not always accurately read the temperature when using a singleuse heat-sensitive chemical strip. Therefore, it is important that nurses ensure they understand how to read the results of these strips correctly.

Accurate recording of physiological observations is essential if a patient becomes acutely unwell. For example, temperature is an important vital sign in the recognition of patients who have developed an infection. Early recognition of the signs of infection has been reinforced by NICE (2016), which details the potentially life-threatening consequences of infection for patients, such as developing sepsis.

Accuracy is also vital in cases where reducing temperature is used as a treatment method. For example, following a stroke, one clinical target is to reduce temperature to normal or below normal to reduce swelling in the brain (Harris and Andrews 2014). Cooling is also used in the treatment of patients who have experienced cardiac arrest, where it is referred to as 'targeted temperature management' and is aimed at preventing cerebral damage (Vaity et al 2015). Cold infusion (using a rapid infusion of cold saline) as a method of inducing cooling is discussed in the literature relating to cardiac arrest, although there is contradictory evidence as to its effectiveness (Bernard et al 2016, Lindsay et al 2018). Studies have also been undertaken into the effectiveness of cold infusion when used to induce therapeutic hypothermia in stroke patients, although the results were inconclusive (Harris and Andrews 2014).

\section{References}

Barnason S, Williams J, Proehl J et al (2012) Emergency nursing resource: non-invasive temperature measurement in the emergency department. Journal of Emergency Nursing. 38, 6, 523-530. doi: 10.1016/j.jen.2012.05.012.

Bernard SA, Smith K, Finn J et al (2016) Induction of therapeutic hypothermia during out-of-hospital cardiac arrest using a rapid infusion of cold saline: the RINSE trial (rapid infusion of cold normal saline). Circulation. 134, 11, 797-805. doi: 10.1161/CIRCULATIONAHA.116.021989.

Carroll DL (2012) Comparison of Temporal to Pulmonary Artery Temperature Measurement in Patients with Fever. https://clinicaltrials.gov/ct2/show/NCT01503294 (Last accessed: 27 February 2019.)

Chue AL, Moore RL, Cavey A et al (2012) Comparability of tympanic and oral mercury thermometers at high ambient temperatures. BMC Research Notes. 5, 356. doi: 10.1186/1756-0500-5-356.

Creagh-Brown BC, James DA, Jackson SH (2005) The use of the Tempa.Dot thermometer in routine clinical practice. Age and Ageing. 34, 3, 297-299. doi: 10.1093/ageing/afi069.

Davie A, Amoore J (2010) Best practice in the measurement of body temperature. Nursing Standard. 24, 42, 42-49. doi: $10.7748 / \mathrm{ns} 2010.06 .24 .42 .42 . c 7850$.

Faulds M, Meeking T (2013) Temperature management in critically ill patients. Continuing Education in Anaesthesia in Critical Care \& Pain. 13, 3, 75-79. doi: 10.1093/bjaceaccp/mks063.

Gasim GI, Musa IR, Abdien MT et al (2013) Accuracy of tympanic temperature measurement using an infrared tympanic membrane thermometer. BMC Research Notes. 6, 194. doi: 10.1186/1756-0500-6-194.

Grainger A (2013) Principles of temperature monitoring. Nursing Standard. 27, 50, 48-55. doi: 10.7748/ns2013.08.27.50.48.e7242.

Harris BA, Andrews PJD (2014) A lesson on induction of hypothermia and measurement of efficacy. Critical Care. 18, 6, 710. doi: 10.1186/s13054-014-0710-y.

Hillier MD (2015) How to wash your hands effectively. Nursing Standard. 30, 3, 34-36. doi: 10.7748/ns.30.3.34.e9691. 
Lindsay PJ, Buell D, Scales DC (2018) The efficacy and safety of prehospital cooling after out-of-hospital cardiac arrest: a systematic review and meta-analysis. Critical Care. 22, 66. doi: 10.1186/s13054-018-1984-2.

National Institute for Health and Care Excellence (2007) Acutely III Adults in Hospital: Recognising and Responding to Deterioration. Clinical guideline No. 50. NICE, London.

National Institute for Health and Care Excellence (2016) Sepsis: Recognition, Diagnosis and Early Management. Clinical guideline No. 51. NICE, London.

Resuscitation Council (UK) (2019) Guidelines and Guidance: The ABCDE Approach. www.resus.org.uk/resuscitation-guidelines/abcdeapproach (Last accessed: 28 February 2019.)

Royal College of Physicians (2017) National Early Warning Score (NEWS) 2: Standardising the Assessment of Acute-Illness Severity in the NHS. RCP, London.

Sund-Levander M, Grodzinsky E (2013) Assessment of body temperature measurement options. British Journal of Nursing. 22, 16, 942 944-950. doi: 10.12968/bjon.2013.22.16.942.

Vaity C, Al-Subaie N, Cecconi M (2015) Cooling techniques for targeted temperature management post-cardiac arrest. Critical Care. 19 , 1, 103. doi: 10.1186/s13054-015-0804-1. 